\title{
Pandemia de COVID-19 e os cuidados de enfermagem aos pacientes em tratamento hemodialítico
}

COVID-19 pandemic and nursing care for patients in hemodialysis treatment La pandemia de COVID-19 y los cuidados de enfermería para pacientes sometidos a hemodiálises

Bernadete Marinho Bara De Martin

Gama $^{1}$ (1)

Carla Mariana Alves da $\mathrm{Cruz}^{2}$ (1) Ludmilla Mursa de França² (D) Mariana Ramalho Ferreira ${ }^{2}$ (1)

Sarah Simões Gomes ${ }^{2}$ (1) Marluce Rodrigues Godinho ${ }^{1}$ (B)

1. Universidade Federal de Juiz de Fora, Faculdade de Enfermagem. Juiz de Fora, MG, Brasil.

2. Universidade Federal de Juiz de Fora, Faculdade de Enfermagem, Curso de graduação em Enfermagem, Juiz de Fora, MG, Brasil.
Autor correspondente: Marluce Rodrigues Godinho Email: marlucerodriguesenf@gmail.com

Recebido em 13/10/2020.

Aprovado em 18/11/2020.

\section{Resumo}

Objetivo: refletir sobre os cuidados de enfermagem aos pacientes em hemodiálise no contexto da pandemia de COVID-19. Método: trata-se de um estudo reflexivo, realizado mediante análise de documentos oficiais dos órgãos de saúde, artigos científicos e outras fontes conceituadas. Está organizado nos seguintes eixos: Pandemia de COVID-19; Insuficiência Renal Crônica e o tratamento hemodialítico; e Cuidados de enfermagem aos pacientes em tratamento hemodialítico no contexto da COVID-19. Resultado: as ações de educação em saúde, educação continuada e a supervisão em enfermagem ganharam destaque no contexto da pandemia. Elas garantiram as orientações necessárias aos pacientes e familiares e à equipe de enfermagem, para prevenção e controle da COVID-19. Consequentemente, contribuíram para a proteção da saúde dos pacientes com insuficiência renal crônica, que já apresentavam sua saúde comprometida e não poderiam deixar de realizar a hemodiálise. Considerações finais e implicações para a prática: no contexto da pandemia da COVID-19, os profissionais de enfermagem precisam redobrar a atenção na assistência prestada aos pacientes em tratamento hemodialítico, além de adaptarem-se às novas orientações. Espera-se que esta reflexão contribua para que os cuidados de enfermagem sejam os mais seguros possíveis, tanto para pacientes e familiares quanto para os profissionais de enfermagem.

Palavras-chave: Insuficiência Renal Crônica; Assistência ao Paciente; Pandemias; Diálise Renal. Cuidados de Enfermagem.

\section{Abstract}

Objective: to reflect on nursing care for hemodialysis patients in the context of the COVID-19 pandemic. Method: This is a reflective study carried out through the analysis of official documents from health agencies, scientific articles and other reputable sources, organized in the following axes: Pandemic of COVID-19; Chronic kidney failure and hemodialysis treatment and Nursing care for patients undergoing hemodialysis in the context of COVID-19. Results: Health education, continuing education and nursing supervision have gained prominence in the context of the pandemic. They have provided the necessary guidance to patients and family members and to the nursing team, for prevention and control of COVID-19. Consequently, they contributed to the health protection of patients with chronic kidney failure who already had their health compromised and could not fail to perform hemodialysis. Final considerations and implications for practice: In the context of the COVID-19 pandemic, nursing professionals need to redouble their attention in assisting patients in hemodialysis treatment, in addition to adapting to the new guidelines. It is hoped that this reflection will contribute to the safest possible nursing care for patients and families and for nursing professionals.

Keywords: Chronic Kidney Failure; Patient Care; Pandemics; Renal Dialysis; Nursing Care.

\section{Resumen}

Objetivo: Reflexionar sobre el cuidado de enfermería de los pacientes en hemodiálisis en el contexto de la pandemia COVID-19. Método: Se trata de un estudio reflexivo realizado a través del análisis de documentos oficiales de organismos de salud, artículos científicos y otras fuentes acreditadas, organizado en los siguientes ejes: Pandemia de COVID-19; Insuficiencia rena crónica y el tratamiento de hemodiálisis y Atención de enfermería a pacientes en hemodiálisis en el contexto del COVID-19. Resultados: Se pudo reflexionar que las acciones de educación en salud, educación continua y supervisión de enfermería cobraron protagonismo en el contexto de la pandemia, a fin de garantizar la orientación necesaria a los pacientes y familiares y al personal de enfermería, para la prevención y control del COVID-19. Consecuentemente contribuyeron a la protección de la salud de los pacientes con insuficiencia renal crónica que ya tienen su salud comprometida y no pueden dejar de someterse a hemodiálisis. Consideraciones finales e implicaciones para la práctica: En el contexto de la pandemia de COVID-19, los profesionales de enfermería necesitan redoblar su atención en la atención brindada a los pacientes en hemodiálisis, además de adaptarse a las nuevas pautas. Se espera que esta reflexión contribuya a que el cuidado de enfermería sea lo más seguro posible, tanto para los pacientes y familiares como para los profesionales de enfermería.

Palabras clave: Insuficiencia Renal Crónica; Atención al Paciente; Pandemias; Diálisis Renal; Atención de Enfermeira. 


\section{INTRODUÇÃO}

O novo coronavírus - denominado Severe Acute Respiratory Syndrome Coronavirus 2 (SARS-CoV-2), causador da doença Coronavirus Disease 2019 (COVID-19) - , ${ }^{1}$ originou-se em Wuhan, na China, mais precisamente em dezembro de 2019, após relatados casos de pneumonia com etiologia desconhecida em moradores da região. ${ }^{2}$ Em janeiro de 2020, a Organização Mundial da Saúde (OMS) comprovou a presença e disseminação do novo vírus. Ao final desse mesmo mês, anunciou a epidemia como uma emergência internacional, visto que diferentes países já registravam casos confirmados, como Estados Unidos, Canadá e Austrália. ${ }^{3-5}$

No Brasil, os primeiros casos suspeitos começaram a ser investigados no início de fevereiro de 2020, mas ainda sem registros de confirmação. ${ }^{3} \mathrm{Em}$ março, com o avanço da doença, a OMS decretou estado de pandemia, após a marca de 118.319 casos e 4.292 mortes ao redor do mundo. ${ }^{6}$ Até o final de agosto, dentre os 25 milhões de casos confirmados e 852.758 mortes no mundo, somente no Brasil, já haviam sido registrados quase 4 milhões de casos confirmados e 121.381 mortes. $^{7}$

OSARS-CoV-2 tem potencial de transmissão por gotículas respiratórias e contato próximo com pessoas infectadas. Devido à sua alta transmissibilidade, são necessários cuidados essenciais, como a higienização das mãos com água e sabão ou álcool em gel a 70\%, o uso de máscaras e, principalmente, o distanciamento social. ${ }^{8,9}$ Embora as informações relativas ao mecanismo de ação do novo coronavírus ainda estejam sendo estudadas, pesquisas já apontaram que pessoas com doenças crônicas, como a Insuficiência Renal Crônica (IRC), fazem parte do grupo de maior risco. ${ }^{10,11}$

Além disso, estudos mostraram que a Hipertensão Arterial Sistêmica (HAS) e o Diabetes Mellitus (DM) são fatores de risco, tanto para a IRC quanto para a ocorrência de complicações à saúde, em consequência da COVID-19. Pessoas que apresentam HAS são mais vulneráveis ao SARS-CoV-2, pois ele pode afetar o músculo do coração, e acredita-se que o vírus infecte células por meio da Enzima Conversora de Angiotensina 2 (ECA2), que possui um papel vital nos sistemas cardiovascular e imunológico, embora os mecanismos específicos sejam ainda incertos. ${ }^{12}$ No DM, os indivíduos apresentam uma baixa na imunidade, devido ao descontrole metabólico ligado à elevação do açúcar no sangue, além do possível sobrepeso. ${ }^{13}$

Essa confluência de fatores de risco torna os pacientes com IRC mais vulneráveis à COVID-19, e suas complicações mais severas, uma vez que o risco de agravamento relaciona-se a maior idade e tempo de duração da doença e o estado do controle metabólico, podendo levar a óbito. ${ }^{13}$ É importante ressaltar que os pacientes com IRC apresentam controle metabólico prejudicado, porquanto os rins tornam-se ineficientes para realizar o processo de filtração do sangue ${ }^{14}$ necessitando de uma Terapia Renal Substitutiva (TRS), sendo mais utilizada a hemodiálise (HD). ${ }^{15,16}$

Nos serviços de hemodiálise, especificamente, há uma preocupação maior na prevenção do contágio pelo vírus, principalmente devido ao fato de o tratamento hemodialítico ocorrer em salas coletivas, ${ }^{17}$ com clientes vindos de diferentes lugares, e estes estão em contato com outras pessoas. Assim, visando frear a contaminação pelo vírus, os órgãos governamentais se empenharam na criação de políticas e diretrizes de boas práticas, e estabeleceram orientações específicas para os profissionais de saúde. ${ }^{18}$

A partir de então, tornou-se necessário adequar-se às novas políticas e medidas preventivas e controle de infecção, tanto pelas unidades e profissionais de saúde quanto pelos pacientes e familiares/acompanhantes, para evitar ou diminuir ao máximo a transmissão do SARS-CoV- $2 .{ }^{18}$ Diante do exposto, emergiram indagações sobre o cuidar dos pacientes renais crônicos em cenários de pandemias, sendo produzido este manuscrito, cujo objetivo é refletir sobre os cuidados de enfermagem aos pacientes em tratamento hemodialítico no contexto da pandemia de COVID-19.

\section{MÉTODO}

Este é um artigo de reflexão, realizado a partir da leitura crítica de documentos oficiais dos órgãos de saúde, artigos científicos e outras fontes conceituadas. A seleção dos materiais de leitura foi realizada de abril a outubro de 2020, nos portais Biblioteca Virtual em Saúde (BVS) e CAPES Periódicos. Utilizaram-se ainda legislações e diretrizes publicadas pelo Ministério da Saúde do Brasil, Centers for Disease Control and Prevention (CDC), Agência Nacional de Vigilância Sanitária (ANVISA), Sociedade Brasileira de Nefrologia (SBN), Organização Mundial da Saúde (OMS) e Organização Pan-Americana da Saúde (OPAS).

\section{RESULTADOS E DISCUSSÃO}

Após a leitura dos materiais selecionados, para embasar a reflexão sobre os cuidados de enfermagem aos pacientes em tratamento hemodialítico, no contexto da COVID-19, primeiramente se fez necessário maior detalhamento acerca de tal contexto, a IRC e o tratamento hemodialítico.

\section{Pandemia de COVID-19}

O epicentro da COVID-19 ocorreu em um mercado atacadista de frutos do mar e animais vivos, o Huanan Seafood Market, em Wuhan, província de Hubei, no sul da China, no fim de 2019. Em 2020, o vírus havia se disseminado por centenas de países e provocado inúmeros óbitos, fazendo com que medidas de biossegurança - como precaução por contato e aerossóis fossem adotadas, a fim de reduzir os riscos de transmissão. ${ }^{2,8,19}$

No Brasil, o primeiro caso foi registrado em São Paulo, em fevereiro de 2020, e a primeira morte em março, na mesma cidade, sendo nesse mesmo mês declarada a transmissão comunitária em nível nacional. ${ }^{13,20,21} \mathrm{Em}$ menos de seis meses, somavam-se quase 4 milhões de casos confirmados e cerca de 120 mil mortes no país. ${ }^{20}$

Até agosto de 2020, os possíveis sintomas da COVID-19 caracterizavam-se como: tosse, febre, coriza, dor de garganta, dificuldade respiratória, cansaço, diminuição do apetite, 
distúrbios gastrointestinais e perda do paladar e olfato. ${ }^{22}$ Os sintomas se apresentam de forma mais branda ou mais grave, independentemente da idade da pessoa infectada. Por isso, deve-se observar a ocorrência de sintomas mais severos, como problemas respiratórios, dor no peito, confusão mental e lábios e rosto azulados, pois, nessas circunstâncias, é recomendado buscar atendimento médico imediato. ${ }^{23}$ Dentre as complicações comuns, destacam-se a Síndrome Respiratória Aguda Grave (SRAG), arritmias, isquemia miocárdica, miocardite, choque, pneumonia, insuficiência renal, bem como a ocorrência do óbito. ${ }^{8,13,24}$

Com base nas informações atuais disponíveis, foram considerados os grupos de risco para o desenvolvimento das formas mais graves da COVID-19: idosos, crianças, gestantes, puérperas, tabagistas, cardiopatas, obesos, diabéticos, asmáticos, pessoas com doenças pulmonares crônicas, imunocomprometidos, asilados, moradores em situação de rua, indígenas, privados de liberdade, doentes renais crônicos e em hemodiálise. Portanto, necessitam cumprir rigorosamente as medidas de prevenção, a fim de reduzir os riscos de mortalidade. ${ }^{13}$

Devido ao fato de se alojar nas vias respiratórias, o vírus pode ser transmitido por pessoas infectadas mediante contato próximo - como um aperto de mãos com gotículas de saliva e secreções de tosse e espirros - e, também, por meio de objetos e superfícies com a presença do microrganismo - celulares, mesas, brinquedos, utensílios domésticos, estetoscópios e termômetros. ${ }^{9,22}$ Em outubro de 2020, os pesquisadores descobriram ainda a possibilidade de transmissão pelo ar, por meio de aerossóis, que podem ficar em suspensão por horas, em locais fechados com ventilação inadequada como restaurantes e academias de ginástica. Nesses casos, o vírus pode ser transmitido a indivíduos que estão até mais afastados fisicamente da pessoa infectada. ${ }^{25,26}$

Outra questão pertinente é a transmissão pré-sintomática, que ocorre quando o portador do vírus não apresenta sintomas, mas possui potencial para a sua propagação, visto que o período de incubação varia, em média, do $5 .^{\circ}$ até o $14 .^{\circ}$ dia de infecção. ${ }^{27,28}$

O diagnóstico da infecção se pauta na pesquisa clínica, no cenário epidemiológico e nos exames de imagem e laboratoriais, ${ }^{22}$ como a sorologia, o teste rápido e o Reverse Transcription Polymerase Chain Reaction(RT-PCR). Dos três, o RT-PCR é considerado padrão-ouro, pois identifica a presença do vírus no trato respiratório, detectando a infecção em estágio agudo e contribuindo para confirmação diagnóstica de indivíduos sintomáticos ou assintomáticos. ${ }^{1}$ Daí a importância da testagem em massa, que possibilita a identificação precoce de infectados e a recomendação de isolamento, visando a redução da transmissibilidade e a realização da vigilância epidemiológica de maneira mais efetiva.

Vale ressaltar que isolamento social é a separação de pessoas doentes ou infectadas, para evitar a transmissão e a disseminação da doença. Por sua vez, a quarentena está relacionada a restringir atividades ou separar pessoas que não estão doentes, mas que possam ter sido expostas à doença ou agente infeccioso, com o objetivo de monitoramento de sintomas e detecção precoce de casos. ${ }^{29}$ Qualquer pessoa em quarentena que desenvolve doença febril ou sintomas respiratórios deve ser tratada como um caso suspeito de COVID-19 e, assim, redobrar a atenção no que diz respeito à prática da higiene respiratória/ etiqueta da tosse..$^{18,29}$

Diante da alta transmissibilidade do SARS-CoV-2, diversos órgãos governamentais e de saúde se empenham na criação de diretrizes que reforcem as medidas de segurança e contenham o seu avanço. Dentre as principais medidas propostas, são frequentemente estimulados o isolamento social, a higienização das mãos com água e sabão ou álcool em gel 70\%, e a incorporação das máscaras no cotidiano da população. 8,9

Além disso, os serviços públicos e privados também necessitam de adequações, principalmente quando é impossível identificar a origem da contaminação, sendo a transmissão, nesse caso, classificada como "transmissão comunitária". Assim, os gestores estaduais e municipais devem estar atentos para adaptar as orientações de acordo com a realidade local. Algumas das medidas preventivas incluem a vacinação contra a gripe, a redução de deslocamentos para o trabalho e mudanças de comportamento e rotina, com a adoção de horários alternativos, a fim de evitar aglomerações. ${ }^{30}$

Quanto àqueles que não têm a possibilidade de permanecer em casa e adaptar o trabalho para ser realizado em suas residências, é recomendado que, ao utilizar o transporte público, sigam as recomendações necessárias de acordo com a Associação Nacional de Empresas de Transportes Urbanos: o uso contínuo de máscara dentro dos transportes públicos; a redução de passageiros; o reforço da limpeza e higienização dos ônibus, terminais e pontos; e a dispensação de álcool em gel a $70 \%$ para passageiros e funcionários. ${ }^{31}$ É importante também manter as janelas abertas durante a circulação, fixar cartazes explicativos sobre a pandemia, em locais de fácil visibilidade para os passageiros, e a disponibilização de Equipamentos de Proteção Individual (EPI) aos funcionários. ${ }^{31}$

\section{Insuficiência Renal Crônica e o tratamento hemodialítico}

A IRC é uma doença crônica não transmissível, que se enquadra em um contexto de suma importância no âmbito da Saúde Pública. No Brasil, há um crescimento progressivo de sua incidência, devido, especialmente, ao potencial aumento da expectativa de vida da população e à presença de comorbidades como a HAS, o DM e o sedentarismo. ${ }^{32}$

A IRC caracteriza-se pela insuficiência dos rins, que, devido a diversos fatores e patologias, torna-se ineficaz em seu processo de filtração, bem como na sua capacidade de manter o equilíbrio hidroeletrolítico, acumulando elementos tóxicos e nocivos ao corpo. ${ }^{14}$ Por consequência das inúmeras complicações sistêmicas e da funcionalidade renal afetada, todo o organismo sofre alterações, tornando-se indispensável, em seu estágio terminal, uma Terapia Renal Substitutiva (TRS) - como a HD, a diálise peritoneal ou o transplante renal -, a fim de equilibrar os distúrbios causados no organismo e a progressão da doença. ${ }^{15}$ 
A HD é uma das TRS mais comuns, realizada por cerca de $90 \%$ dos pacientes em tratamento dialítico registrados em 2019 , no Brasil, ${ }^{16}$ modalidade geralmente persistente por toda vida, uma vez que o transplante renal não é disponível e recomendado para todos os pacientes. ${ }^{33}$

Os indivíduos em diálise renal ou tratamento hemodialítico podem apresentar inúmeras modificações em suas vidas, assim como na rotina das pessoas com quem convivem, devido aos impactos no âmbito psicossocial e nas questões do próprio tratamento, por exemplo, a constância e manutenção dessa terapia. Os transtornos perpassam desde o desconforto físico e os efeitos colaterais das medicações, até as mudanças na alimentação, infecções e menor qualidade de vida, ${ }^{34}$ além de uma considerável diminuição da força muscular respiratória. ${ }^{35}$

Tendo em vista todos os desafios enfrentados e as diversas transformações em seu estilo de vida e hábitos, torna-se fundamental o apoio familiar juntamente com a equipe multiprofissional, no sentido de estimular a adaptação ao tratamento, bem como no enfrentamento das mudanças decorrentes da IRC e seus agravantes ${ }^{36}$ Vale ressaltar que a IRC possui efeitos degenerativos nos diversos sistemas do corpo - entre eles o sistema imunológico -, colocando o paciente renal crônico no grupo de risco no que diz respeito aos cuidados preventivos quanto à COVID-19. ${ }^{37}$

Considerando o impacto na Saúde Pública e na vida das pessoas - tanto em virtude da pandemia da COVID-19 quanto da IRC e do tratamento hemodialítico -, infere-se que merece uma atenção especial a discussão sobre a assistência de enfermagem ao paciente com IRC que realiza diálise renal. Ou seja, faz-se necessário reforçar os cuidados específicos com esses indivíduos, os quais já apresentam a saúde debilitada e risco maior de desenvolver complicações relacionadas à infecção pelo novo coronavírus.

\section{Cuidados de enfermagem aos pacientes em tratamento hemodialítico no contexto da COVID-19}

No serviço de diálise, o enfermeiro é responsável pelo gerenciamento do cuidado, atuando na organização e direção dos serviços de enfermagem e suas atividades técnicas e auxiliares, tendo compromisso com o paciente e a instituição quanto à qualidade da assistência prestada, embasado no Código de Ética dos Profissionais de Enfermagem e na Lei do Exercício Profissional da Enfermagem. ${ }^{38-40}$ Assim, o cuidado de enfermagem é indispensável no cotidiano dos pacientes em TRS, e os profissionais precisam estar constantemente atentos às atualizações sobre o uso correto dos EPI, o rigor na assistência prestada e as medidas de prevenção de agravos, como vem acontecendo no contexto da pandemia da COVID-19, reconhecendo sinais e sintomas da doença, para que a disseminação do vírus seja contida, especialmente para pacientes renais. ${ }^{18,41}$

O enfermeiro, juntamente à equipe de enfermagem, deve: reorganizar o fluxo de pessoas, orientando pacientes e acompanhantes a evitarem circular em áreas comuns desnecessariamente, bem como o compartilhamento de alimentos ou objetos pessoais; aconselhar a presença de acompanhantes somente em casos de extrema necessidade; orientar pacientes e familiares a realizarem contato telefônico com o serviço de diálise, em caso de aparecimento de sinais e sintomas da doença ou tenha tido contato com terceiros suspeitos/confirmados com o vírus; além de promover a educação em saúde ao fornecer máscaras, incentivar e orientar pacientes e acompanhantes quanto ao uso correto durante toda a permanência no serviço de diálise. . $^{14,18,42,43}$

$\mathrm{O}$ enfermeiro deve ainda: eleger salas individuais para dialisar pacientes suspeitos e/ou confirmados com a COVID-19; realizar o descarte das linhas e dialisadores utilizados naqueles sob suspeita ou confirmação da doença, após cada sessão de hemodiálise; e garantir o envio de amostras de água e sangue para análise laboratorial. ${ }^{18,42,43}$

Entre as recomendações específicas às unidades de diálise, destacam-se também: garantir a continuidade do tratamento dialítico a pacientes sob suspeita ou confirmação diagnóstica; definir estratégias para reduzir a disseminação de microrganismos no ambiente e um fluxo distinto para os pacientes sintomáticos/ suspeitos; orientar os pacientes e familiares a realizarem contato telefônico imediato, no caso de surgimento de sinais e sintomas ou de contato próximo com pessoas sob suspeita/confirmação diagnóstica da doença; aplicar questionários para pesquisar a possível presença de sintomas respiratórios; afixar cartazes com orientações que auxiliem na compreensão das medidas de higiene a serem adotadas; e oferecer local adequado para a higienização das mãos, reduzindo ao máximo o contato direto com torneiras e lixeiras. ${ }^{18,42,43}$

Tornou-se importante ainda: disponibilizar suprimentos próximos às poltronas dos pacientes, para estimular a constante higienização das mãos; definir produtos para saúde próprios aos pacientes suspeitos ou confirmados; realizar limpeza e desinfecção rigorosa em máquinas, mobílias e equipamentos após as sessões; avaliar a possibilidade do atendimento em domicílio para os pacientes que testaram positivo, e direcionar profissionais exclusivamente a essa função; além de respeitar a distância mínima de um metro entre as cadeiras e estimular medidas educativas para os profissionais que apresentaram sintomas e, por isso, necessitaram ser afastados do trabalho. ${ }^{18}$

Diante do cenário da pandemia - que trouxe a necessidade de intensas adaptações -, vale reforçar o direito do enfermeiro de aprimorar seus conhecimentos técnico-científicos visando fundamentar a prática profissional, de maneira a oferecer assistência de qualidade e com segurança aos pacientes e para si. Ao mesmo tempo, esse é um dever do profissional, uma vez que tal aprimoramento refere-se ao benefício da pessoa, família e coletividade em qualquer circunstância. ${ }^{44}$

Outro aspecto que merece atenção especial refere-se ao fato de que a pandemia da COVID-19 encontra-se inserida em um contexto no qual a tecnologia está presente de variadas formas. Ademais, a assistência ao paciente demanda adaptações e medidas de segurança mais rígidas, muitas pessoas sentem necessidade de registrar tudo que acontece utilizando seus dispositivos móveis. Porém, o Código de Ética dos Profissionais de Enfermagem assegura-lhes o direito de negarem-se a ser 
filmados, fotografados ou expostos em mídias sociais durante o desempenho de suas atividades. Essa exposição transgride o direito constitucional do brasileiro e o assegurado aos profissionais de enfermagem à privacidade, além de provocar certa vulnerabilidade para eles e os pacientes, pois as informações expostas podem ser interpretadas de diversas maneiras. ${ }^{44,45}$

Portanto, neste momento de intensas transformações e incertezas, destaca-se a importância das ações de educação em saúde, educação continuada e supervisão em enfermagem - enquanto atividades essenciais desenvolvidas pelo enfermeiro-, consolidando seu compromisso ao oferecer as orientações necessárias aos pacientes e familiares/acompanhantes. Além disso, ao capacitar e supervisionar a equipe de enfermagem, para o adequado cumprimento das ações de prevenção e controle da COVID-19, favorecem a proteção da saúde dos pacientes em tratamento de hemodiálise.

\section{CONSIDERAÇÕES FINAIS E IMPLICAÇÕES PARA A PRÁTICA}

Após a discussão e reflexão realizadas, é possível perceber que são inúmeros os desafios enfrentados pelos serviços de saúde e suas equipes em todo o mundo. Os profissionais de enfermagem, que apresentam contato direto e ininterrupto com pacientes, familiares e acompanhantes, ao longo de sua jornada de trabalho, precisam redobrar sua atenção para reduzir as chances de infecção para si e para os demais que não estejam com o vírus. Dessa forma, faz-se necessário que os serviços de saúde, em especial os de diálise, mantenham-se sempre atualizados diante das constantes e rápidas descobertas e modificações sobre o manejo do novo coronavírus, visando prevenir ao máximo que os pacientes com IRC, que já apresentam a saúde fragilizada, desenvolvam a doença e, possivelmente, sofram com as suas complicações.

É importante ressaltar que, em contextos anteriores à pandemia, os cuidados de enfermagem aos pacientes em tratamento hemodialítico eram pautados em ações voltadas à prevenção de complicações, tendo em vista a gravidade da IRC para o equilíbrio biopsíquico e social dos indivíduos. Anterior à pandemia, já eram empregados protocolos cuidadosamente detalhados, antes, durante e após as sessões de hemodiálise. Com o seu advento, ocorreu maior rigor na atenção e no desenvolvimento das ações realizadas e a adaptação às novas orientações, no sentido de prevenir, não só o contágio pelos microrganismos já existentes, mas também pelo SARS-CoV-2, devido ao seu potencial de agravamento nesses pacientes em especial.

Nessa direção, espera-se que o presente estudo possibilite melhor compreensão sobre as mudanças na operacionalização dos cuidados de enfermagem aos pacientes em hemodiálise, no cenário de pandemia, mediante legislações determinadas pelo governo e novas orientações de entidades de saúde. Além disso, é nesses momentos de desafios que o compromisso social da enfermagem ganha maior visibilidade quando, ao integrar a equipe de saúde multi e interdisciplinar, atua na linha de frente no combate ao SARS-CoV-2, consolidando seu valor e competência para a realização de cuidado integral ao paciente, família e coletividade com excelência, ética e profissionalismo.

Vale enfatizar que a principal limitação deste estudo está relacionada ao fato de que as orientações fornecidas foram fundamentadas de acordo com informações disponíveis sobre a pandemia, em documentos oficiais dos órgãos de saúde do governo, artigos científicos e demais fontes conceituadas. Tais informações podem sofrer mudanças e atualizações de acordo com as novas demandas que venham surgir e a realização de novos estudos. Porém, essas orientações, juntamente às medidas de prevenção e controle durante a assistência, contribuirão para minimizar a disseminação da COVID-19 e também desenvolver um novo olhar para a relevância dos cuidados de enfermagem. Por fim, espera-se que o produto gerado a partir desta reflexão possibilite a transformação do conhecimento em instrumento prático, a fim de que os cuidados de enfermagem sejam os mais seguros possíveis, tanto para os pacientes com IRC quanto para seus familiares/acompanhantes e os próprios profissionais de enfermagem.

\section{CONTRIBUIÇÕES DOS AUTORES}

Desenho do estudo de reflexão: Bernadete Marinho Bara de Martin Gama. Carla Mariana Alves da Cruz. Ludmilla Mursa de França. Mariana Ramalho Ferreira. Sarah Simões Gomes. Marluce Rodrigues Godinho.

Levantamento de informações: Bernadete Marinho Bara de Martin Gama. Carla Mariana Alves da Cruz. Ludmilla Mursa de França. Mariana Ramalho Ferreira. Sarah Simões Gomes. Marluce Rodrigues Godinho.

Análise reflexiva: Bernadete Marinho Bara de Martin Gama. Carla Mariana Alves da Cruz. Ludmilla Mursa de França. Mariana Ramalho Ferreira. Sarah Simões Gomes. Marluce Rodrigues Godinho.

Redação e revisão crítica do manuscrito: Bernadete Marinho Bara de Martin Gama. Carla Mariana Alves da Cruz. Ludmilla Mursa de França. Mariana Ramalho Ferreira. Sarah Simões Gomes. Marluce Rodrigues Godinho.

Aprovação da versão final do artigo: Bernadete Marinho Bara de Martin Gama. Carla Mariana Alves da Cruz. Ludmilla Mursa de França. Mariana Ramalho Ferreira. Sarah Simões Gomes. Marluce Rodrigues Godinho.

Responsabilidade por todos os aspectos do conteúdo e a integridade do artigo publicado: Bernadete Marinho Bara de Martin Gama. Carla Mariana Alves da Cruz. Ludmilla Mursa de França. Mariana Ramalho Ferreira. Sarah Simões Gomes. Marluce Rodrigues Godinho.

\section{EDITOR ASSOCIADO}

Antonio José de Almeida Filho 


\section{REFERENCES}

1. Ministério da Saúde (BR). CORONAVÍRUS COVID-19 - Acurácia dos testes diagnósticos registrados na ANVISA para a COVID-19 [Internet] Brasília: Ministério da Saúde; 2020 [citado 2020 jul 13]. Disponível em: https://portalarquivos2.saude.gov.br/images/pdf/2020/June/02/ AcuraciaDiagnostico-COVID19-atualizacaoC.pdf

2. World Health Organization. Novel Coronavirus (2019-nCoV): situation report - 1 [Internet]. Geneva:WHO; 2020 [citado 2020 ago 16]. Disponível em: https://www.who.int/docs/default-source/coronaviruse/situationreports/20200121-sitrep-1-2019-ncov.pdf?sfvrsn=20a99c10_4

3. Ministério da Saúde (BR), Secretaria de Vigilância em Saúde. Infecção Humana pelo Novo Coronavírus (2019-nCoV). Boletim Epidemiológico. 2020;2:1-23.

4. World Health Organization. IHR Procedures concerning public health emergencies of international concern (PHEIC) [Internet]. Geneva: WHO; 2005 [citado 2020 dez 8]. Disponível em: https://www.who.int/ ihr/procedures/pheic/en/

5. World Health Organization. Novel Coronavirus (2019-nCoV): Situation Report - 11 [Internet]. Geneva:WHO;2020 [citado 2020 dez 7]. Disponível em: https://www.who.int/docs/default-source/coronaviruse/situationreports/20200131-sitrep-11-ncov.pdf?sfvrsn=de7c0f7_4

6. World Health Organization. Coronavirus disease 2019 (COVID-19): Situation Report - 51 [Internet]. Geneva: WHO; 2020 [citado 2020 ago 16]. p. 1-9. Disponível em: https://www.who.int/docs/defaultsource/coronaviruse/situation-reports/20200311-sitrep-51-covid-19. pdf?sfvrsn=1ba62e57_10

7. World Health Organization. WHO Coronavirus Disease (COVID-19) Dashboard [Internet]. Geneva: WHO; 2020 [citado 2020 ago 31]. Disponivel em: https://covid19.who.int/

8. Ministério da Saúde (BR), Secretaria de Atenção Especializada à Saúde, Departamento de Atenção Hospitalar, Domiciliar e de Urgência. Protocolo de manejo clínico da Covid-19 na Atenção Especializada [Internet]. Brasília: Ministério da Saúde; 2020 [citado 2020 jul 15] Disponível em: https://portaldeboaspraticas.iff.fiocruz.br/wp-content/ uploads/2020/04/manejo_clinico_covid-19 atencao especializada. pdf

9. Organização Pan-Americana da Saúde, Organização Mundial da Saúde. Orientação sobre o uso de máscaras no contexto da COVID-19 [Internet]. 2020 [citado 2020 jul 20]. Disponível em: https://iris.paho.org/ bitstream/handle/10665.2/52254/OPASWBRACOVID-1920071_por. pdf? sequence $=1$ \&isAllowed $=y$

10. Ministério da Saúde (BR). Diretrizes para diagnóstico e tratamento da COVID-19 [Internet]. Brasília: Ministério da Saúde;2020 [citado 2020 ago 18]. Disponivel em: http://docs.bvsalud.org/biblioref/2020/05/1096254/ diretriz-covid19-v4-07-0520h05m.pdf

11. Liu W, Li H. COVID-19: attacks the 1-beta chain of hemoglobin and captures the porphyrin to inhibit heme metabolism. Chem Rxiv. [Internet] 2020 [citado 2020 ago 28]. Disponível em: https://chemrxiv.org/articles/ COVID-19_Disease_ORF8_and_Surface_Glycoprotein_Inhibit_Heme_ Metabolism_by_Binding_to_Porphyrin/11938173/6

12. Zheng Y-Y, Ma Y-T, Zhang J-Y, Xie X. COVID-19 and the cardiovascular system. Nat Rev Cardiol. 2020;17(5):259-60. https://doi.org/10.1038/ s41569-020-0360-5.

13. Conselho Nacional de Secretarias Municipais de Saúde, Conselho Nacional de Secretários de Saúde. Guia Orientador para o enfrentamento da pandemia Covid-19 na Rede de Atenção à Saúde [Internet]. Brasília: CONASEMS / CONASS; 2020 [citado 2020 ago 18]. Disponível em: https://www.conasems.org.br/wp-content/uploads/2020/05/InstrumentoOrientador-Conass-Conasems-VERS\%C3\%83O-FINAL-3.pdf

14. Lemos Horta HH, Lopes ML. Complicações decorrentes do tratamento dialítico: contribuição do enfermeiro no cuidado e educação ao paciente. Rev Enferm Contemp. 2017 out 30;6(2):221. http://dx.doi. org/10.17267/2317-3378rec.v6i2.1457.

15. Marques BM, Silva DM, Roseira CE, Orlandy FS, Figueiredo RM. Análise de indicadores de qualidade e características clínicas em uma unidade de terapia renal substitutiva. Cuid Enferm [Internet]. 2019; [citado 2020 jul 18];13(2):99-105. Disponível em: http://www.webfipa.net/facfipa/ner/ sumarios/cuidarte/2019v2/99.pdf
16. Sociedade Brasileira de Nefrologia. Censo de diálise SBN. São Paulo: SBN; 2019. p. 50.

17. Arenas MD, Villar J, González C, Cao H, Collado S, Crespo M et al. Manejo de la epidemia por coronavirus SARS-CoV-2 (COVID-19) en unidades de hemodiálisis. Nefrologia. 2020 maio;40(3):258-64. http:// dx.doi.org/10.1016/j.nefro.2020.04.001. PMid:32340751.

18. Agência Nacional de Vigilância Sanitária (BR). Nota Técnica GVIMS/ GGTES/ANVISA No 04/2020. Orientações para serviços de saúde: medidas de prevenção e controle que devem ser adotadas durante a assistência aos casos suspeitos ou confirmados de infecção pelo novo coronavírus (SARS-CoV-2) [Internet]. Brasília: ANVISA; 2020 [citado 2020 maio 8]. Disponível em: http://portal.anvisa.gov.br/ documents/33852/271858/Nota+T\%C3\%A9cnica+n+04-2020+GVIMSGGTES-ANVISA/ab598660-3de4-4f14-8e6f-b9341c196b28

19. World Health Organization. WHO advice for international travel and trade in relation to the outbreak of pneumonia caused by a new coronavirus in China [Internet]. Geneva:WHO; 2020 [citado 2020 jul 28]. Disponíve em: https://www.who.int/news-room/articles-detail/who-advice-forinternational-travel-and-trade-in-relation-to-the-outbreak-of-pneumoniacaused-by-a-new-coronavirus-in-china

20. Ministério da Saúde (BR). COVID-19 - Painel Coronavírus [Internet]. Brasília: Ministério da Saúde; 2020 [citado 2020 ago 31]. Disponíve em: https://covid.saude.gov.br/

21. Ministério da Saúde (BR). Ministério da Saúde declara transmissão comunitária nacional [Internet]. Brasília: Ministério da Saúde; 2020 [citado 2020 jul 19]. Disponível em: https://www.saude.gov.br/noticias/agenciasaude/46568-ministerio-da-saude-declara-transmissao-comunitarianacional\#: :text=0\%20Minist\%C3\%A9rio\%20da\%20Sa\%C3\%BAde\%20 declarou,se\%20unir\%20contra\%200\%20v\%C3\%ADrus

22. Ministério da Saúde (BR). COVID-19 - Sobre a doença [Internet] Brasília: Ministério da Saúde; 2020 [citado 2020 ago 15]. Disponível em: https://coronavirus.saude.gov.br/sobre-a-doenca\#sintomas

23. Centers for Disease Control and Prevention. Coronavirus disease 2019 (COVID-19): symptoms of coronavirus [Internet]. 2020 [citado 2020 maio 20]. Disponível em: https://www.cdc.gov/coronavirus/2019-ncov/ symptoms-testing/symptoms.html

24. Adami ER, Imig DC, Ribas JLC. COVID 19: revisão, relato de caso e perspectivas. Rev UNIANDRADE. 2020;21(1):36-48. http://dx.doi. org/10.5935/1519-5694.20200004.

25. Centers for Disease Control and Prevention. Coronavirus Disease 2019 (COVID-19): your health - how it spreads [Internet]. 2020 [citado 2020 nov 16]. Disponível em: https://www.cdc.gov/coronavirus/2019-ncov/ prevent-getting-sick/how-covid-spreads.html

26. Prather KA, Marr LC, Schooley RT, McDiarmid MA, Wilson ME, Milton DK. Airborne transmission of SARS-CoV-2. Science. 2020 out 16;370(6514):303-4. http://dx.doi.org/10.1126/science.abf0521. PMid:33020250.

27. Lauer SA, Grantz KH, Bi Q, Jones FK, Zheng Q, Meredith HR et al The incubation period of coronavirus disease 2019 (covid-19) from publicly reported confirmed cases: estimation and application. Ann Intern Med. 2020 maio 5;172(9):577-82. http://dx.doi.org/10.7326/ M20-0504. PMid:32150748.

28. Yu P, Zhu J, Zhang Z, Han Y. A Familial Cluster of Infection Associated With the 2019 novel coronavirus indicating possible person-toperson transmission during the incubation period. J Infect Dis. 2020 maio 11;221(11):1757-61. http://dx.doi.org/10.1093/infdis/jiaa077. PMid:32067043.

29. Organização Pan-Americana da Saúde, Organização Mundial da Saúde. Considerações para quarentena de indivíduos no contexto de contenção da doença causada pelo novo coronavírus (COVID-19) [Internet]. 2020 [citado 2020 jul 29]. Disponível em: https://iris.paho. org/bitstream/handle/10665.2/51956/OPASBRACOVID1920018_por. pdf? sequence $=1$ \&isAllowed=y

30. Ministério da Saúde (BR). Saúde anuncia orientações para evitar a disseminação do coronavírus [Internet]. Brasília: Ministério da Saúde; 2020 [citado 2020 ago 16]. Disponível em: https://www.saude.gov.br/ noticias/agencia-saude/46540-saude-anuncia-orientacoes-para-evitara-disseminacao-do-coronavirus 
31. Associação Nacional das Empresas de Transportes Urbanos. COVID-19 e o transporte público por ônibus: impactos no setor e ações realizadas [Internet]. Brasília: Associação Nacional das Empresas de Transportes Urbanos; 2020 [citado 2020 abr 20]. Disponível em: https://www.ntu. org.br/novo/upload/Publicacao/Pub637231535674949776.pdf

32. Castro RVRS, Rocha RLP, Araujo BFM, Prado KF, Carvalho TFS. Chronic renal patient perception on experience in hemodialysis. Rev Enferm Cent-Oeste Min. 2018 set 10;8. http://dx.doi.org/10.19175/ recom.v8i0.2487.

33. Santos MVB, Lira GG, Fernandes FECV. Medication adherence by the chronic renal patient on hemodialysis. Rev Enferm UFPE online. 2020;14:1-8. https://doi.org/10.5205/1981-8963.2020.243294.

34. Santos GLC, Alves TF, Quadros DCR, Giorgi MDM, Paula DM. The person's perception about its condition as a chronic renal patient in hemodialysis. Rev Pesqui Cuid É Fundam Online. 2020 jun 1;636-41. http://dx.doi.org/10.9789/2175-5361.rpcfo.v12.9086.

35. Dorneles PP, Ferrareze ME, Carpes M, Lemos FA, Bueno AF, Veronese FV et al. Força muscular respiratória e capacidade funcional em pacientes com doença renal crônica. Arq Ciênc Saúde UNIPAR. 2019 dez 4;23(3):203-8. http://dx.doi.org/10.1590/S1517-86922010000400002.

36. Fidelis $\mathrm{Cl}$, Balbino CM, Tavares e Souza MM, Rodrigues LMS, Silvino ZR, Passos JP. Dificuldades enfrentadas pelo paciente renal para a realização do tratamento. Rev Enferm Atual Derme. 2016;77(15):16-21. http://dx.doi.org/10.31011/reaid-2016-v.77-n.15-art.369.

37. Silva AC, Souza ATS, Arenas VG, Barros LFNM. A ação do enfermeiro na prevenção de doenças renais crônicas: uma revisão integrativa. SANARE [Internet]. 2015 jul; [citado 2020 ago 14];14(2):148-55. Disponível em: https://sanare.emnuvens.com.br/sanare/article/view/840/511

38. Freitas JS, Silva AEBC, Minamisava R, Bezerra ALQ, Sousa MRG Quality of nursing care and satisfaction of patients attended at a teaching hospital. Rev Lat Am Enfermagem. 2014 jun;22(3):454-60. http://dx.doi. org/10.1590/0104-1169.3241.2437. PMid:25029057.
39. Pennafort VP, Furtado AM, Fialho AV, Moreira TM, de Freitas MC Queiroz MV. Produção do conhecimento científico de Enfermagem em Nefrologia. Rev Bras Enferm. 2010 out;63(5):830-6. http://dx.doi. org/10.1590/S0034-71672010000500022. PMid:21103780.

40. Carvalho IMP, Melo RL, Andraus LMS. Produção científica de enfermagem em nefrologia, no Brasil, no período de 1989 até 1999. Rev Eletrônica Enferm [Internet]. 2006 dez;3(2). http://dx.doi.org/10.5216/ree.v3i2.724.

41. Silva AS, Silveira RS, Fernandes GFM, Lunardi VL, Backes VMS Percepções e mudanças na qualidade de vida de pacientes submetidos à hemodiálise. Rev Bras Enferm. 2011 out;64(5):839-44. http://dx.doi. org/10.1590/S0034-71672011000500006. PMid:22460483.

42. Núcleo de Vigilância Sanitária/SRS Uberaba. VISA e COVID-19 Orientações Gerais - Versão 2 - 18/04/2020 [Internet]. 2020 [citado 2020 ago 18]. Disponível em: https://www.ipatinga.mg.gov.br/abrir_arquivo. aspx/Orientacoes_sobre_COVID_18042020?cdLocal=2\&arquivo=\%7 BDC3258E7-AEED-6B25-2347-64DDB111B1BD\%7D.pdf

43. Sociedade Brasileira de Nefrologia. Protocolo de orientação para colegas que cuidem de pacientes portadores de doenças renais raras em virtude da epidemia da SARS COV 2 (Severe Acute Respiratory Syndrome, CoronaVirus - 2) [Internet]. São Paulo: SBN; 2020 [citado 2020 ago 20]. Disponível em: https://www.sbn.org.br/fileadmin/diversos/ novo_doencas_raras.pdf

44. Resolução COFEN No 564/2017 (BR). Aprova o novo Código de Ética dos Profissionais de Enfermagem. Diário Oficial da União [periódico na internet], Brasília (DF), 2017 [citado 2020 ago 23]. Disponível em: http://www.cofen.gov.br/resolucao-cofen-no-5642017_59145.html

45. Senado Federal (BR). Constituição da República Federativa do Brasi de 1988: promulgada em 5 de outubro de 1988. Diário Oficial da União [periódico na internet], Brasília (DF), 1988 [citado 2020 maio 10]. Disponível em: http://www.planalto.gov.br/ccivil_03/constituicao/ constituicao.htm 